\title{
WildSense: Monitoring Interactions among Wild Deer in Harsh Outdoor Environments Using a Delay-Tolerant WSN
}

\author{
Junho Ahn, ${ }^{1}$ Akshay Mysore, ${ }^{1}$ Kati Zybko, ${ }^{2}$ Caroline Krumm, ${ }^{2}$ Sravan Thokala, ${ }^{1}$ \\ Xinyu Xing, ${ }^{3}$ Ming Lian, ${ }^{1}$ Richard Han, ${ }^{1}$ Shivakant Mishra, ${ }^{1}$ and Thompson Hobbs ${ }^{2}$ \\ ${ }^{1}$ Department of Computer Science, University of Colorado, Boulder, CO 80309, USA \\ ${ }^{2}$ Natural Resource Ecology Laboratory, Department of Ecology, Colorado State University, Fort Collins, CO, USA \\ ${ }^{3}$ Georgia Institute of Technology, Department of Computer Science, Atlanta, GA, USA \\ Correspondence should be addressed to Junho Ahn; junho.ahn@colorado.edu
}

Received 26 May 2016; Accepted 13 July 2016

Academic Editor: Xiaobei Zhang

Copyright (C) 2016 Junho Ahn et al. This is an open access article distributed under the Creative Commons Attribution License, which permits unrestricted use, distribution, and reproduction in any medium, provided the original work is properly cited.

\begin{abstract}
Biologists and ecologists often monitor the spread of disease among deer in the wild by using tracking systems that record their movement patterns, locations, and interaction behavior. The existing commercial systems for monitoring wild deer utilize collars with GPS sensors, deployed on captured and rereleased deer. The GPS sensors record location data every few hours, enabling researchers to approximate the interaction behavior of tracked deer with their GPS locations. However, the coarse granularity of periodically recorded GPS location data provides only limited precision for determining deer interaction behavior. We have designed a novel system to monitor wild deer interaction behavior more precisely in harsh wilderness environments. Our system combines the functionalities of both GPS and RF-radio sensors with low-cost and minimal-resource motes. We designed and built our system to be able to operate robustly for a period of up to several months for continual tracking and monitoring of the locations and interaction behaviors of wild deer in harsh environments. We successfully deployed six deer collars on six wild deer that were captured and rereleased in the Soapstone Prairie Natural Area of northern Colorado over a one-month period. In this paper, we describe how we designed and built this system and evaluate its successful operation in a wilderness area.
\end{abstract}

\section{Introduction}

The development of systems for remote tracking of animals represents the single most influential advance in instrumentation for observing ecological processes in populations and communities of vertebrates. Until this development, it was impossible to consistently observe animals in their natural habitats except under the most idiosyncratic conditions. Despite its wide use, current technology for these systems suffers from three fundamental limitations. First, existing low-cost and low-performance wireless sensors are highly sensitive and can easily malfunction in harsh outdoor environments (e.g., high humidity, strong winds, heavy dust or mud, and drastic temperature changes). Second, costs of implementing telemetry constrain sample sizes, leading to undesirable limitations on statistical power. It follows that there is a need for instruments that allow spatially accurate observations at costs that are one-tenth of the costs of current telemetry systems. Third, current instrumentation is "location centric" only-it allows the investigator to know the position of the individual animal relative to some geographic position at some specified time but cannot inform questions about its frequent interactions with other individuals. It follows that there is a need for remote tracking technology that can better record accurate interactions among animals.

This paper describes the design, implementation, and evaluation of WildSense, a software and hardware system that allows scientists to collect location and contact information of free-range deer in a low cost and robust manner in a harsh wilderness environment, as shown in Figure 1. WildSense is comprised of collar nodes and a delay-tolerant network for relaying contact information between radio-based collar nodes. The collar node shown is worn by each animal being monitored and consists of a MICAz mote (equipped with a 


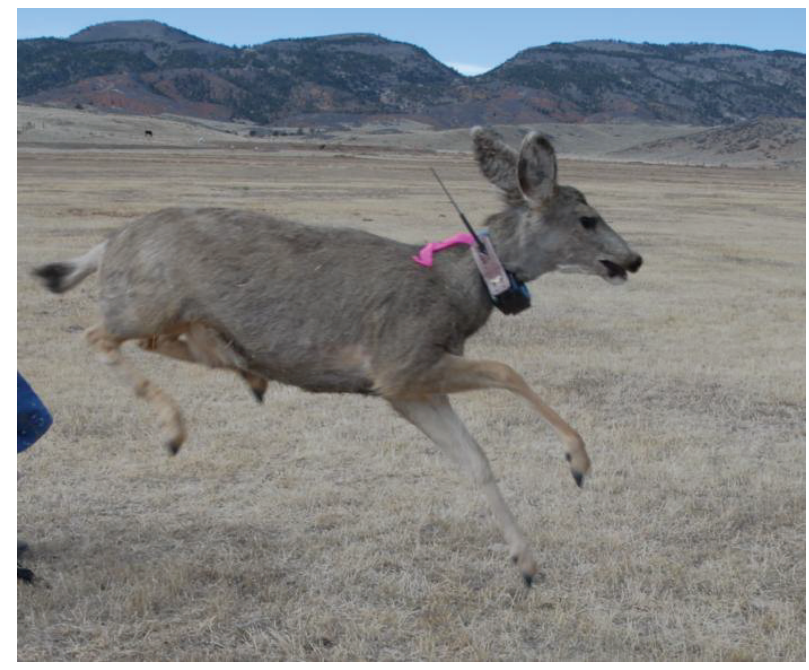

Figure 1: Collar deployment for wild deer.

low-power short range IEEE 802.15.4 2.4 GHz radio, TinyOS, and a GPS receiver) and also a VHF transmitter. WildSense employs a special type of networking protocol called a delaytolerant network (DTN) to route information between nodes. Such a network is robust to disconnection between nodes in the network, allowing nodes to exchange each other's information when in close proximity, while storing information for future exchanges when nodes are out of range. As a result, DTNs are ideally suited for exchanging information among mobile wildlife that may only intermittently be in contact.

WildSense is designed for sustained low-cost deployment in rugged wilderness areas. It is designed to last more than 45 days without requiring any battery recharging, so that it is sufficiently weather/terrain independent. It is also designed to tolerate failures that may occur in real world deployments. For example, the DTN protocol has the property that it improves the fault tolerance of the communication network. Location and interaction information is automatically replicated and propagated in the network, so that the loss of any one collar node will still allow a subset of that animal's contact information to be retrieved from other nodes in the network. WildSense is also designed to be a low-cost system, where each collar costs less than $\$ 450$ in total. This paper presents detailed results from a successful deployment of WildSense on six free-range mule deer, for a period of one month, in the Soapstone Prairie Natural Area near Livermore, Colorado.

This paper makes the following key research contributions to enable improved tracking of wild animals in rugged, harsh outdoor environments. First, our understanding is that the results of this paper provide some of the most detailed validation to date of DTN protocols in practice, particularly how their fault tolerance properties enable effective operation in harsh real world environments. We show that wild deer coming in contact with one another enables the forwarding and spread of contact information via a DTN protocol, thereby providing robustness to failures; that is, when a collar node was lost, its contact information was preserved in the information exchanged with other DTN nodes on each contact. This robustness property of DTN protocols, successfully highlighted by the WildSense deployment's results, improves the ability of scientists to recover an increased amount of data from their field experiments. Second, WildSense shows the feasibility of sampling free-range animal interactions in a manner that is both fine grained and low powered. In comparison, the current approach using GPS collars is faced with a tradeoff: if finer grained location sampling is desired, then longevity is hampered due to the power-hungry GPS unit operating for an increased time; conversely, if longevity is desired, then the sampling granularity is forced to be more coarse grained. WildSense provides the ability to collect the frequency of contacts down to the minute granularity, while also providing long-duration observations.

The remainder of this paper is organized as follows. Section 2 provides an overview of related work. Section 3 describes the details of the software and hardware design of WildSense. Section 4 describes the details of the WildSense deployment in Soapstone Prairie Natural Area for a period of about 30 days, as well as the data collection process involved in this deployment. Section 5 provides a detailed evaluation of the data we collected from the deployment of our system on the free-range deer in a wilderness environment. We finish with a discussion and summarize our findings in the conclusion.

\section{Related Work}

Existing ecological research [1-3] that tracked white-tailed deer utilized GPS-based systems to measure their movements and interaction behavior among them (same group and different group). The movement research [1] has shown that deer walk within a home range of 10 kilometers. Mean daily travel distance [2] was 778 meters with a range between 506 and 1500 meters during the excursion duration. Our system 
also utilized the GPS sensor to monitor their movements and we compared the results collected in our system and the existing research results to analyze our system's feasibility. Additionally, although the existing interaction research measured deer behavior in both the same group and different group, it was limited to measuring the deer' locations on an hourly basis to conserve its battery power. The research [3] has found that white-tailed deer interact with others frequently in the same group and very infrequently in a different group by analyzing location data collected hourly. Our WildSense system provides the most precise ability to collect the frequency of contacts down to a granularity of less than one minute of interaction duration with a delay-tolerant WSN.

Though there is extensive literature exploring the topic of delay- and disruption-tolerant networks (DTNs), as summarized in a number of surveys [4-7], the vast majority of these papers are based exclusively on simulation. While simulations can provide helpful insights, they can also be limited by potentially unrealistic assumptions that do not hold true in real world deployments, like unrealistic mobility models, idealized wireless channel models, energy models that are too optimistic, and overly simplistic failure models, to name just a few. For example, Spray and Wait [8] simulate a routing scheme that uses a flooding algorithm for intermittently connected networks where a few packets are sprayed into the network and an acknowledgment is sent when the sprayed packet is received at the sink. This piece of information is then used to improve the performance of the network. Li et al. [9] used trace-based simulation to evaluate a socially selfish algorithm where the node forwards its data only to other nodes with which it has strong ties. Pásztor et al. [10] propose context aware data forwarding so that nodes in the network can calculate an efficient path of transfer to the base station. Code is built for motes, but the protocol is evaluated via a simulator and is not deployed in the field. Routing approaches strongly related to DTN routing include message ferrying [11], also called data muling, and routing in vehicular ad hoc networks (VANETS) [12]. These works are primarily simulations based.

Our focus has been on practically deploying a DTN system in the field, so our contributions concern how to operate a DTN successfully in a harsh real world environment. As a result, our literature review below focuses primarily on the small number of practical deployments of DTN systems that have been reported, where the small number testifies we believe to the inherent difficulty and imposing challenges of successfully deploying DTN systems in the wild. The results of this paper provide we believe some of the most detailed validation to date that DTN protocols can operate effectively in demanding in situ conditions.

In terms of real world DTN deployments, perhaps the most similar to ours is Zebranet [13-16]. In this project, wild zebras were collared in Kenya and their activity was monitored for 10 days. They used GPS for recording location information and VHF communication for flooding the data in their network. Solar energy was used to power the hardware in the collars and a rechargeable battery stored the energy for future use. The Zebranet collar was set to sample
GPS every 8 minutes due to power/longevity constraints. Rather than relying on power-hungry GPS to infer contact information, we instead employ low-power radios with a short range (about $30 \mathrm{~m}$ ) to record contact information. This low-power approach enables both fine-grained sampling of contacts (at the rate of at least once per minute) and long term observation (up to 45 days). GPS sampling at a much lower rate of once per hour was retained to provide timing data and approximate location references to the proximity contacts, which was suitable for the domain scientists. Our design also differed from Zebranet by eliminating solar recharging, which could miss contacts due to insufficient recharging under overcast skies. Indeed, ten of our thirty deployment days were either completely overcast/snowing or partly cloudy. As we show later, our system was able to capture all contacts at the granularity of at least once per minute over a span of thirty days, including a key contact that would have been missed by coarser sampling.

A store and forward DTN-like implementation has been briefly reported [17] wherein cattle act as data ferries to information sinks situated near common watering holes. This was tested only with captive animals and not with wildlife. We employed a similar preliminary phase of testing WildSense on managed cattle in paddocks. While this kind of phased testing is useful, these tests could not fully emulate the actual wildlife deployment. For example, we found that the cattle testing failed to predict a failure mode encountered in the actual deployment, namely, losing a collar node. Carnivore [18] is a disruption-tolerant system deployed on mountain lions, in which nodes forward data to the node which has most recently accessed a sink. One collar of three was recovered, with no contacts reported.

DTN-like data forwarding has also been applied in other scenarios such as transportation and agriculture. Daknet [19] extends internet connectivity to public information kiosks in rural India and Cambodia, in which buses plying on transport routes are fitted with mobile access points to ferry the information from rural areas to cities with better internet connectivity. The Wizzy Digital Courier service employs a similar approach to connect to schools in remote villages in South Africa [20]. An energy harvesting data mule [21] system has also been implemented in which a person cycles around soil moisture sensor nodes to collect data and relay them to an internet gateway, where the data mule is powered by dynamos fixed on the cycle. Some studies have logged location information among buses [22], or Bluetooth-based contact information among mobile devices like PDAs and motes $[23,24]$, in order to develop trace-based simulations for evaluating DTN protocols but do not deploy any DTN forwarding in practice.

Wireless sensor networks (WSNs) have also been applied to track animals in a variety of other scenarios, though these do not employ multihop DTN-like forwarding or ferrying of data among the animals. Rather, these systems typically report directly from a sensing node to a collection point, such as a base station. A WSN was deployed to monitor cattle in a 21-hectare paddock $[25,26]$. The cattle were fitted with collars containing GPS and proximity loggers and the information was relayed to a base station using the 


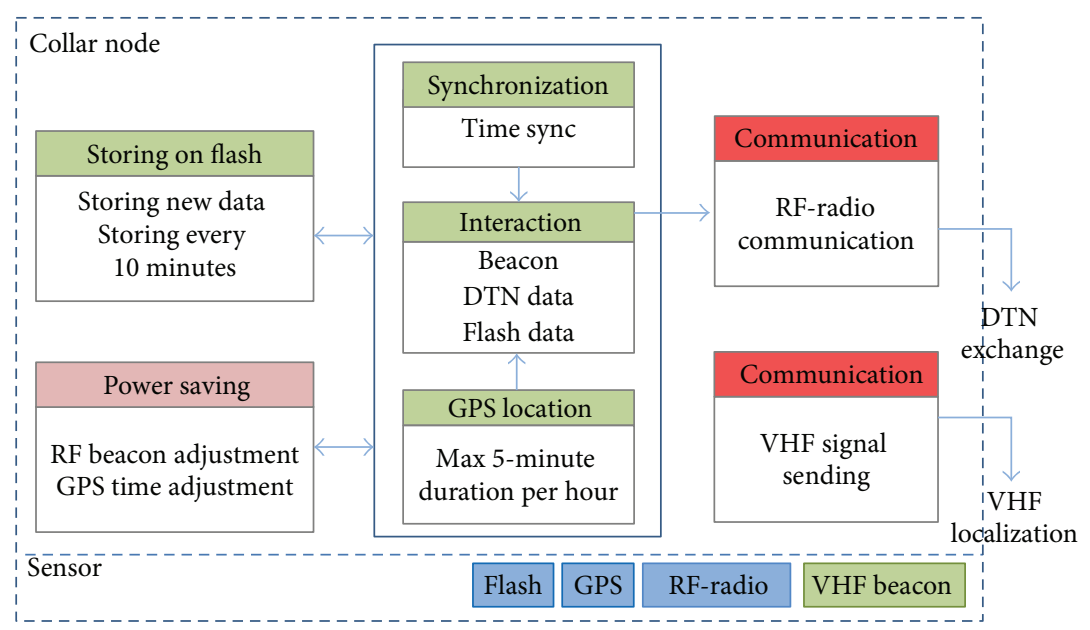

FIgURE 2: System architecture.

reliable and always connected communication network of collars. The velocity and eating habits of cattle were studied [27]. A related electronic shepherd [28] project employed a wireless sensor/actuator network to monitor cattle and control them via mild electric shocks from fighting with each other. Elsewhere, a badger monitoring network was implemented by deploying RFID collars on badgers and fixed RFID sensing stations that transferred the data via $3 \mathrm{G}$ cellular connections [29]. CraneTracker [30] deployed nodes that used cellular communication on whooping cranes to determine their migration pattern and wireless sensor nodes in areas of smaller range, for example, nesting sites. These cellular approaches were not suitable for our deployment, which involved remote terrain that lacked cellular connectivity. wildCense [31] also sought to deploy sensor nodes on deer (in India) but ultimately did not report on any deployments in the field.

\section{System Design}

In this section, we discuss the key aspects of our software and hardware system design. The software must be able to collect and relay contact information with the goal of 45 days of continuous operation, and the hardware must be sufficiently weather proof to endure exposure to the elements in an outdoor area, as well as durable enough for collar deployment on free-range deer.

3.1. Software Design. Our software was designed to track the movement of deer in the wild and especially monitor their interaction, so that scientists can determine contact rates and estimate the rate of disease propagation in wild deer populations, for example, chronic wasting disease. Standard collar nodes used by ecologists track and record the deer's locations based on periodic GPS sampling at the rate of once every few hours to conserve battery power, resulting in a granularity that is too coarse to capture detailed deer interaction behavior. Our software system is designed to capture contact rates at a much finer granularity while operating robustly for extended continuous periods of time.

The deer collar nodes were constructed based on MICAz motes [32], which were desirable for their low cost, low power, light weight, small form factor, RF network and ranging capabilities, software support, and extensibility. We added a GPS module and operated with TinyOS v1.2.3. The size of our software's source code that operated on this hardware was $65.6 \mathrm{~KB}$ out of 128 kilobytes of ROM, utilizing 3905 bytes RAM out of 4012 bytes. Figure 2 shows our system's software architecture.

The collar node collects GPS-based locations and interaction behavior data via low-power RF-radio signals. Collars can be programmed with a timed falloff mechanism. In order to locate a collar that has fallen off and thereby retrieve the collar node and its data, a VHF radio was also installed in each collar node, allowing the collar to be located from its VHF beacon within a maximum $8 \mathrm{~km}$ range. Note that the VHF beacon is strictly used for collecting the collars after they have fallen off. VHF cannot be used for recording contact information that involves exchanging data packets as it uses analog signals.

We describe below each component of our system's software design and how it operates with the collar.

3.1.1. Location. We integrated GPS sensor modules into our deer collar system design for both coarse-grained localization and time synchronization purposes, with the latter motivation explained in more detail below. We tested the GPS sensor in various different types of terrain, such as hills, valleys, streams, forests, and open plains, and found that a safe duration for keeping the GPS sensor on in order for it to acquire a location lock typically took 5 minutes. We also found the safest approach was to reinitialize the GPS sensor periodically, because otherwise it would cease to localize properly. We found if we reinitialized and activated the GPS sensor to run for periods of only up to 5 minutes every hour, we could successfully collect localization data on the 


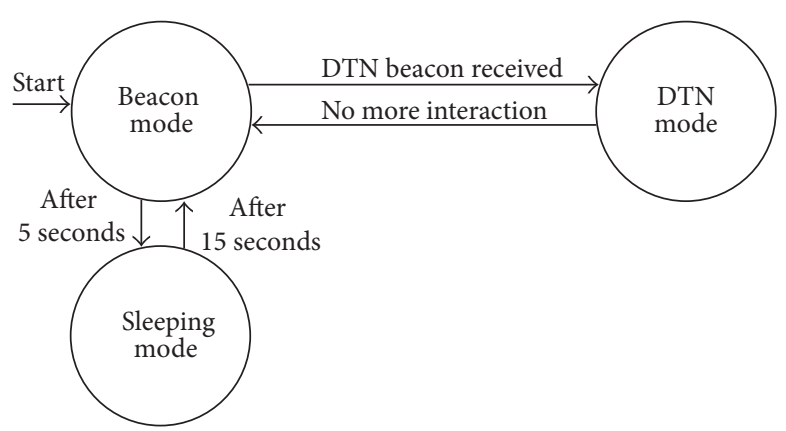

FIGURE 3: State diagram of collar node's software.

deer collar nodes for the duration of the experiment while conserving power via duty cycling.

3.1.2. Interaction. We were able to achieve subminute granularity in terms of capturing contact rates by using MICAz RF-radio communication. The transmit power of the IEEE 802.15.4 radio was set to $0 \mathrm{dBm}$, which gave us a range of approximately $30 \mathrm{~m}$. This was viewed as acceptable by the wildlife biologists to estimate proximity-based contacts for the purposes of this experiment. The radios would beacon by default every 20 seconds, duty cycling to save power by staying up 5 seconds and sleeping 15 seconds. The radios were synchronized to awaken at the same time using GPS time. If a collar node hears another collar node, it drops out of beaconing mode and into DTN mode explained below. These different software states are outlined in Figure 3.

With RF-radio communication, we sought to measure two aspects of deer behavior interaction with our software: the occurrence of a contact interaction event and the duration of such an event. First, when two deer come into close range, each node drops into DTN mode and begins exchanging DTN table data with the other node every 6 seconds. The DTN table is cached in RAM for fast access and due to the small size of the RAM stores only the 8 most recent interaction data segments at one time. New interaction data continually overwrite older data segments, based on the interaction time. The interaction data stored on each collar node includes paired collar node information such as the two interacting node's IDs, locations, local times, GPS times, and additional table data. Records of the most recent contacts thus propagate further throughout the DTN hop by hop each time there is an encounter between two deer.

Second, we log the above-described interaction information on the node's flash, so that we can later assess interaction time duration for the paired nodes.

3.1.3. Synchronization. We needed mechanisms to synchronize the duty cycles of our GPS and low-power radio, as well as time-stamp our interaction data. We utilized two forms of time synchronization, one based on GPS to synchronize our radios for contact information and another based on ETA [33] to measure time differences and thus time-stamp the data by day. First, in order to synchronize the beacon signals and activate the RF-radio sensors at the same time, we based our algorithm on GPS to the second granularity. We found, however, that based on GPS seconds, there could be up to a 3 -second time delay in the sensor nodes receiving the GPS location data. For this reason, we set the radio beacon signals activation time to extend up to 5 seconds during collar node interaction periods and sent two beacon signals at the 2nd and 4th seconds in each 5 -second activation period, rather than one beacon. Using the two beacon signals allowed us to ensure that, within the 20-second activation and sleeping period, we could hear at least one of the RF-radio sensor beacons among interacting collar nodes.

In order to time-stamp the data over the duration of the experiment, which spanned days and weeks of elapsed time, we used a time differencing algorithm based on local time synchronization similar to ETA for wireless sensor networking. We did not use GPS time for time-stamping because the GPS time includes only hours, minutes, and seconds and does not include any date information (year, month, and day). For purposes of our experiment, we needed to track date information to determine where and when the interaction among the deer collar nodes occurred. The ETA algorithm synchronizes node times in milliseconds. It uses local node time to calculate the time difference between a current and past event and then sends this information in packets of 4 bytes each to a neighbor node. We implemented our ETA-style local time synchronization at the application layer.

Pseudocodes 1 and 2 outline the pseudocodes for GPS localization, interaction and packet exchange between nodes, and synchronization of duty cycles of GPS and low-power radio.

3.1.4. Storage on Flash. We wished to permanently store the interaction data and durably log other parameters of the experiment and used flash memory for these purposes. Whenever data is stored on the flash, it is stored in 64-byte chunks. The total storage space of the flash is 512 kilobytes, which can hold 8000 units of such data. Once the flash space is full, the oldest data is continually overwritten by the newest data, using circular buffering. Our software stores both the deer collar node GPS location and the interaction data on flash. Each collar node records GPS location sensor data 


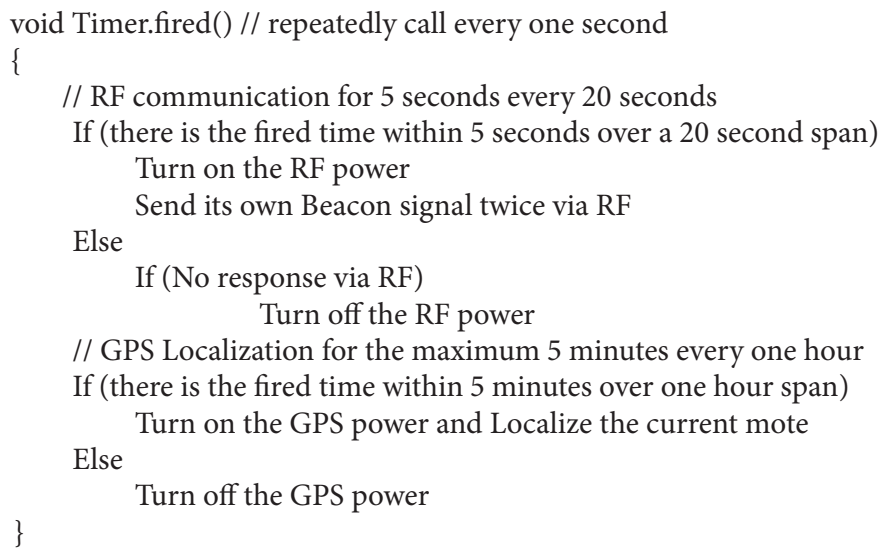

Pseudocode 1: Pseudocode for the timer to send beacon via RF and localize the mote using GPS.

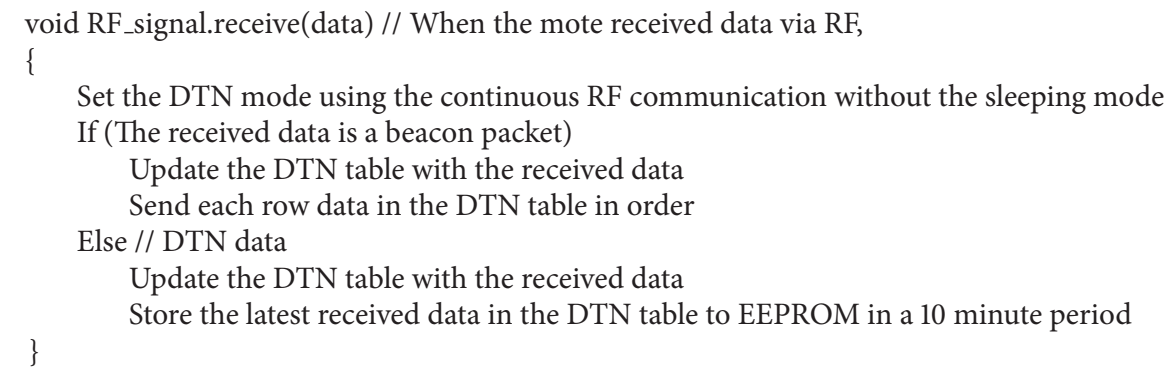

Pseudocode 2: Pseudocode for the RF-receiving part when a mote receives a beacon or DTN data via RF.

directly on the flash every hour. For DTN data, as we noted, data is exchanged and stored every 6 seconds when a contact event is detected. However, if two deer are in continuous contact, as we saw in this deployment, then this could fill the flash quickly with redundant data, when what we really want is duration of contact information. To economize on memory for long-duration contacts, we compare the latest exchanged DTN information, and if it is identical to the previous exchange, we do not store the latest exchange.

While implementing the flash storage functionality, we discovered a complication that caused a crashing problem. Whenever we activated the GPS sensor and data was attempting to store on the flash, both the GPS sensor and the flash crashed. At this point, the flash would no longer store any more data. To solve this problem, we implemented a flag in the source code to check whether the GPS sensor was activating or not, and if it was, we suspended the flash storing functionality until the GPS sensor was finished activating and localizing. Once we had done this, there were no further problems with our flash storage functionality.

3.1.5. Power Saving. In order to save power, both the GPS and the radio are duty cycled, with the GPS at a 1/12 duty cycle and the radio at a $1 / 4$ duty cycle. In addition, we implemented an optimization that shut off our GPS sensors before the full 5 minutes elapsed if they were able to acquire 5 satellites sooner. This could be determined by polling the GPS sensor.

We measured the power consumption of our deer collar nodes when operating in each of four different modes: GPS + RF-radio, only GPS, only RF-radio, and sleeping. For each mode, we measured the amperage and then calculated the power consumption in each of the four modes, based on a voltage of 3.3. For each mode of operation, respectively, we found that our collar node consumed the following amount of battery power: $306.9 \mathrm{~mW}$ in GPS + RF-radio mode; $300.3 \mathrm{~mW}$ in only GPS mode; $75.9 \mathrm{~mW}$ in only RF-radio mode; $0.30 \mathrm{~mW}$ in sleeping mode.

We also investigated the length of time our collar node operated on battery power, with and without all of our power saving approaches. We found that with our algorithms we could extend our sensor node battery operating time up to three times longer than without power savings. For example, in a test with four $1.6 \mathrm{~V} / 7500 \mathrm{mah} \mathrm{C}$-cell batteries, the sensor node operated only 12 days without power savings, but with our power saving techniques it operated for a total of 33 days. However, this approach had insufficient longevity; so ultimately we settled on a design incorporating two paralleled D-cell $3.6 \mathrm{~V}$ batteries with 19,000 mah per each, with a $3.3 \mathrm{~V}$ regulator, and tests showed that it exceeded our 45day lifetime goal. We explain the need for the regulator in 


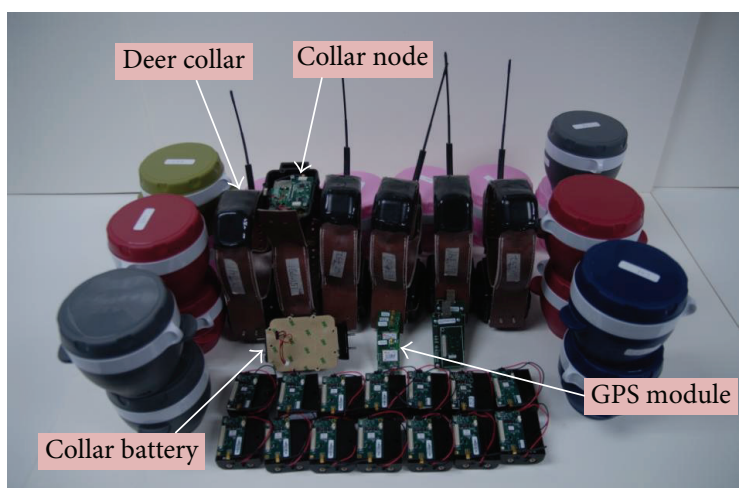

Figure 4: Hardware setup.

Section 3.2. We constructed our deer collar nodes based on these findings.

Second, we recovered the deer collar nodes that had detached from the deer's necks and fallen on the ground. We implemented a dump program to download all of the flash data to a computer and installed this program on the recovered collar nodes. The dump program sent the collar node's flash data every one second through a serial port on the computer.

3.1.6. VHF Beacon. In each deployed deer collar node, we also installed a VHF beacon board that emitted low level, minimal battery power usage signals. These signals are used for locating the collars after they have been dropped off the deer. The VHF beacon board emits two signals every second, but while the collar remained on the deer's neck only one signal could be detected, likely due to its movement and the deer's body and fur absorbing the signal. However, once the collars detached and fell to the ground, both signals emitted by the VHF beacon could be detected within an $8 \mathrm{~km}$ range. To retrieve the detached deer collars that had fallen to the ground and collect our data, we used a VHF receiver and antenna to search for the emitting VHF signals and locate the collar nodes.

3.2. Hardware Design. For our experiment, we assembled and deployed 6 nodes on deer collars in the outdoor prairie wilderness area. Figure 4 shows the hardware equipment used in our experiment. The equipment consisted of $20 \mathrm{MICAz}$ motes, six GPS modules, six GPS antennas, six regulators, six VHF beacon boards, and six VHF antennas. We designed six weather-proof deer collar nodes, that each included a MICAz mote, a GPS module and antenna, a regulator, and a VHF board and antenna. In the section below, we describe the features of the equipment we used in our design of the deer collar nodes.

The MICAz mote we chose to use for our deer experiment is a $2.4 \mathrm{GHz}$ wireless sensor module (mote) used for lowpower applications. It is based on an ATmegal28L microcontroller with $512 \mathrm{~K}$ bytes of serial flash and a Chipcon CC2420 $\mathrm{RF}$ transceiver. To construct the GPS module, we connected an MTS420 environmental sensor board to the MICAz mote via a 51-pin connector. The MTS420 sensor board [34] is equipped with a GPS chip, with position accuracy of 10 meters and acquisition time (warm) of 33 seconds. The mote and sensor board were powered by two 3.6 V Saft LSH D cells via a Texas instruments TPS 61201 boost converter (the regulator) [35] which provided an output of a constant voltage of 3.3. The converter has an operating input voltage range from $0.3 \mathrm{~V}$ to $5 \mathrm{~V}$, with a maximum $300 \mathrm{~mA}$ output, and draws a quiescent current of less than $55 \mu \mathrm{A}$.

We ended up using parallelized 3.6 voltage batteries with the regulator to provide stable power to our sensor board. Initially, we experimented with using a regulator with two $1.6 \mathrm{~V}$ batteries connected in series but found that the voltage would decrease below $2.7 \mathrm{~V}$, at a level where the motes are not guaranteed to collect data reliably. By operating two 3.6 V Dcell batteries in parallel combined with the regulator, we were able to provide a consistent voltage of $3.3 \mathrm{~V}$ to the sensor node for the duration of the experiment.

The collar nodes were built with the help of ATS (Advanced Telemetry Systems) company [36], which designed the VHF beacon board and the drop-off mechanism. Figure 5 shows the collar node installed with the sensors in a secure and robust assemblage. The VHF beacon board, batteries, and regulator were connected with each other and set in acrylic in the outer shell of the collar to prevent disconnection of wires due to the movement of the hardware within the case. The MICAz mote and the sensor board were secured with screws so that they can be easily removed in case they needed to be reprogrammed and connected to the regulator output. Finally, the shell was sealed with a double sided sealing tape to prevent moisture from seeping in and was screwed in to lock it in place.

We affixed a GPS antenna and VHF beacon antenna at the top of the deer collar and connected these to the collar sensor node as shown in Figure 6. We also installed a mechanism for the collar that was programmed to automatically detach and disconnect the collar construction, so that the collar could fall off the deer's neck, and the collar could later be retrieved. This mechanism was programmed to be released on a specific date by ATS company. 


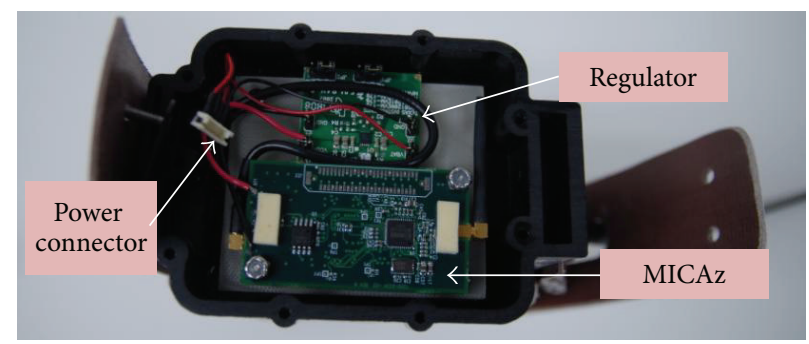

Figure 5: Collar node.
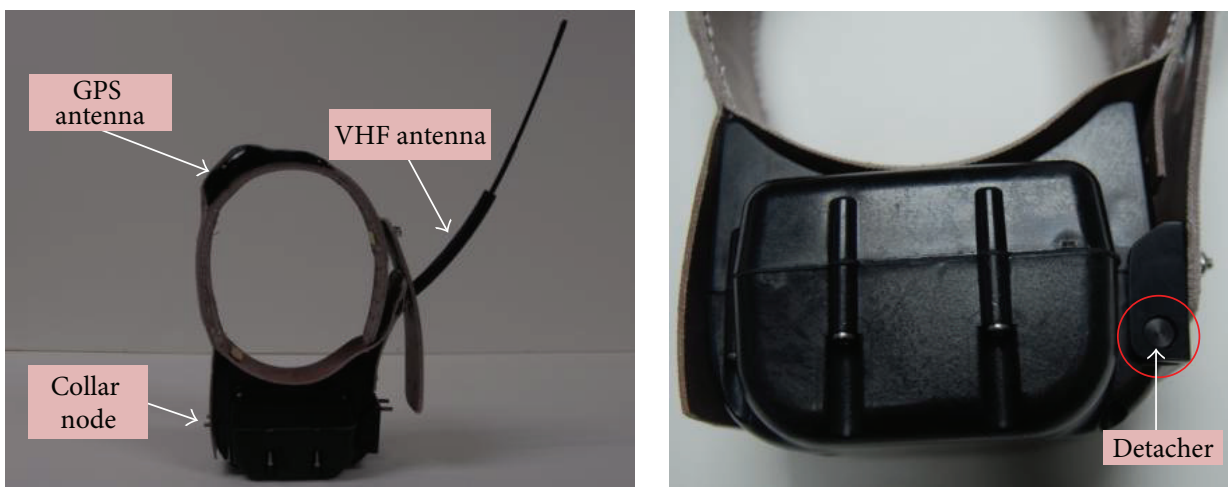

FIgURE 6: Deer collar.

\section{Deployment and Data Collection}

In February 2013, we deployed WildSense over six different deer [37-39] in the Soapstone Prairie Natural Area (a 75.8square-kilometer area) [40] near Livermore, Colorado. The collars were programmed to detach from the deer after approximately three weeks, so that we could retrieve them to collect the sensor data for this research. We describe this deployment process and explain how the collars were retrieved from the outdoor area after the completion of the experiment.

4.1. Collar Node Deployment. Our deployment of collar nodes on wild deer consisted of the following steps: getting permission from appropriate authorities, base camp setup, aerial searching for free-range deer, capture of deer, medical examination and installation of collars on deer, and, finally, the release of deer back into the wilderness. Figure 7 shows an overview of our deer collar deployment process in the wilderness area.

We needed to obtain three different permissions for our wildlife experiment. First, we had to obtain IRB (Institutional Review Board) permission [41] from the federal government to conduct our deer collar deployment experiment. Second, we had to apply to the National Park Service to obtain permission to capture and release deer in this area. Third, because this research was conducted under the auspices of a research institution, each individual involved in the experiment also had to obtain permission to work in the wilderness area on the research team. All individuals involved in this experiment had to be trained on safety precautions by the research team leaders and sign releases, in order to participate in this wildlife experiment. Once all permissions were obtained, we could access the wilderness area to deploy our collars on the deer.

Our research team consisted of ecologists, biologists, a ranger to manage the park, and engineers. The team brought in two helicopters, two fueling trucks for the helicopters, a truck with the medical trailer attached, and six additional trucks to carry the researchers back and forth to the base camp. A small search airplane was also contracted to continually fly over a wide area surrounding the base camp, in search of deer that could then be captured by researchers in the helicopters. Whenever team members caught a free-range deer, the deer were transported by one of the helicopters to the base camp to be medically examined before we installed the collar. We measured the deer's health status, installed the collars on the neck of the deer, and then released the deer back into the wild in the area surrounding the base camp. Figure 8(a) shows the base camp setup we used for deploying our collars on the captured deer. The two helicopter fueling trucks were positioned at a safe distance away from the medical trailer and center of the base camp, to ensure the safety of the research personnel and the deer.

The small search airplane was utilized to locate free-range deer in the large area surrounding the base camp. Once a deer was spotted, the researcher in the plane notified the team at base camp of the deer's location. Then the helicopters were deployed to that location to capture and retrieve the deer. Up to two helicopters were used to capture the deer. The helicopters were manned by two people: a pilot and one of the researchers on the team. Figure 8(b) illustrates how 


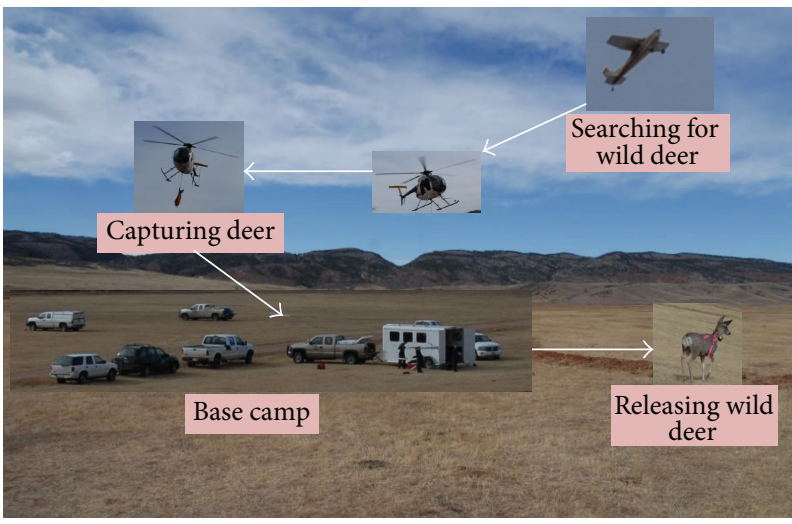

Figure 7: Overview of collar deployment.

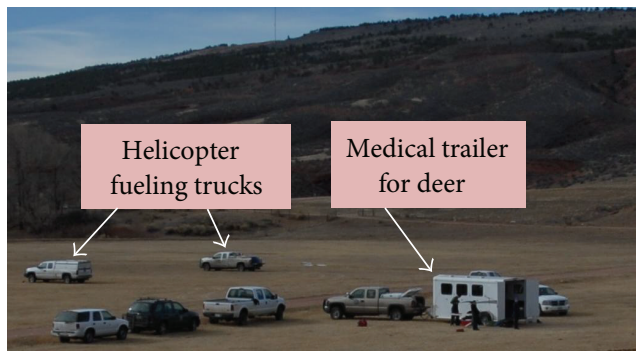

(a)

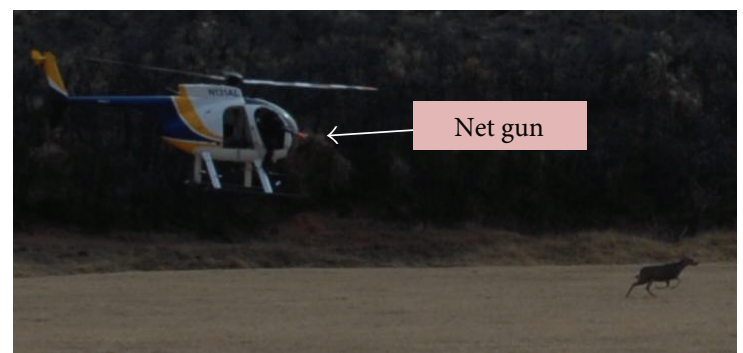

(b)

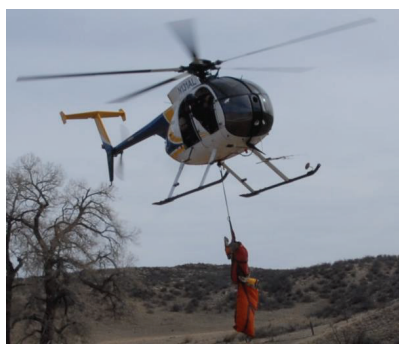

(c)

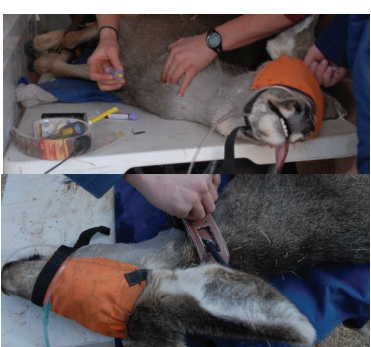

(d)

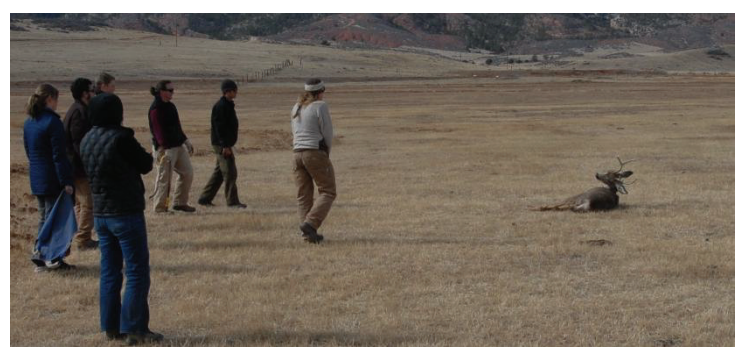

(e)

Figure 8: Steps of collar deployment.

the helicopter research personnel captured the deer using a net gun. As the helicopter approaches a deer, the research personnel were usually able to capture the deer by shooting a net that would entangle the feet of the running deer. At that point, the helicopter would immediately land on the ground, so that the researcher could jump off the helicopter and run to the deer to give it a sleeping drug injection. The researcher then bound the deer's legs and blindfolded the deer. The deer was then placed into a large carrying bag that was attached to and suspended from the helicopter, so it could be transported back to the base camp. Figure 8(c) shows one of the helicopters carrying two captured deer at one time back to base camp.

The captured deer is then delivered to the medical trailer at the base camp in order to allow research personnel to check the health of the deer and collect biological information, before we installed the deer collar. The helicopter dropped off the captured deer at the base camp and four team members carried it into the medical trailer. The deer was given water to prevent dehydration and was provided with oxygen to stabilize it while it was in a drugged state. Figure 8(d) shows the deer being carried into the medical trailer. Biologists did a general checkup and performed tests, such as checking its gender, temperature, and ear passages, and drew blood for further analysis. They also collected a bit of fur and a stool sample for further analysis and did an ultrasound of the female deer to make sure they were not pregnant. They also recorded the unique VHF frequency of the deer collar that was to be used for this particular deer. The collected health status measurements are used to monitor any changes in the deer's health when it is recaptured, at which time new health measurements can be taken and compared to the previous data.

After all the health measurements were taken and the collar was installed, we released the deer back into the wild, at the edge of the base camp area. We untied the rope, still 


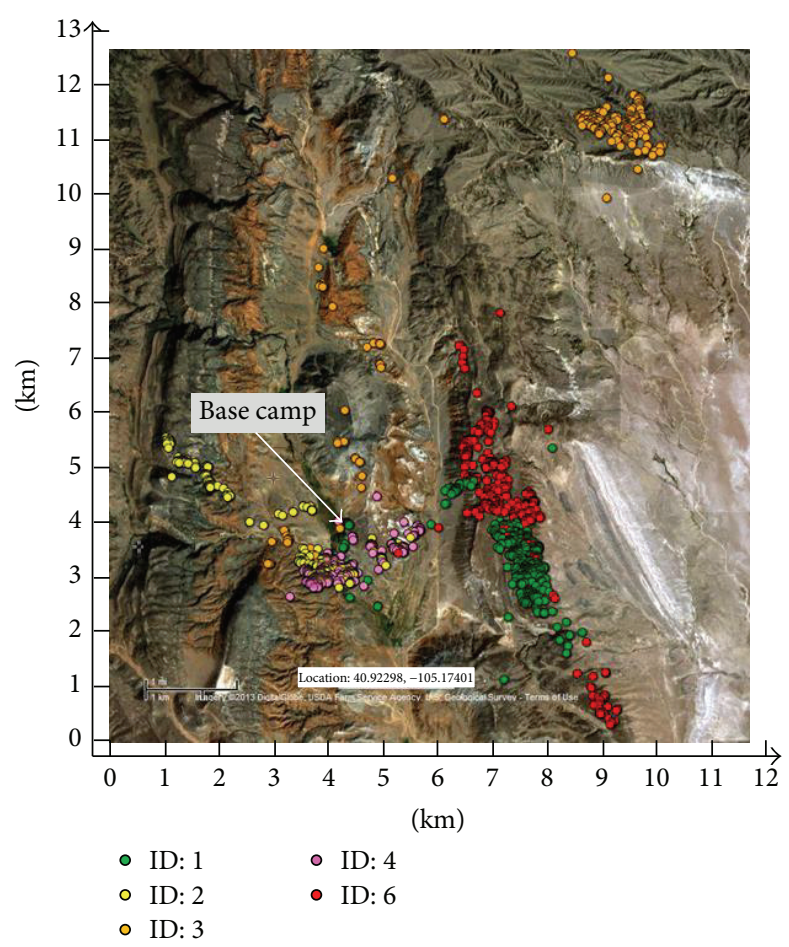

FIGURE 9: GPS tracking of five collected collars on map.

binding the legs, and removed the blindfold from its eyes. Team members stood in a line between the deer and the base camp area, in order to prevent the deer from venturing back to the base camp. We waited until the deer was completely alert, stood up, and ran off into the wild. Figure 8(e) shows the research team releasing the deer back into the wilderness area.

4.2. Collar Collection. We had programmed the collars to automatically detach from the deer's necks after three weeks and began collecting them soon after that, though some of the deer continued carrying the detached collar (draped over their necks) up to a week after that. Team members using a truck with a VHF antenna first located the general area of the dropped-off collar and then walked through the area with a handheld VHF antenna to locate the actual collar. By using this method, we successfully located five of the six collars; one collar's signal was never detected, possibly dropped off in a deep valley or river where we could not detect the signal. We retrieved two of the collars by the beginning of the fourth week, two more collars by the middle of this week, and the fifth collar at the end of the fourth week.

\section{Evaluation}

We have analyzed all GPS location and RF-radio interaction data retrieved from the five recovered collars. Our evaluation focused on analyzing deer movement over time as well as interactions among them. It shows that our system is able to collect detailed information about not only the locations of various deer at different times, but also time, location, and duration of interactions among them. We show that this information can be used to study fine-grained interactions among deer within a social group. Furthermore, our evaluation demonstrates the fault tolerance property of our system, wherein we were able to collect location and movement information of a lost deer as well. Finally, we demonstrate the DTN functionality of our system. We show a detailed view of how location and interaction data was transferred from one deer to another using our DTN protocol over the course of 30 days.

\subsection{Experimental Results for Collar Nodes}

5.1.1. Deer Location and Movement. For clarification, we have assigned IDs from 1 to 6 to the six deer we used in our experiment. Figure 9 shows the locations of five deer whose collars we were able to collect over one-month period. All locations are mapped over the prairie region in which they lived and traveled during the experiment period. Collar nodes collected GPS location data every hour and stored them on flash. Our analysis shows that the deer generally stayed within a foursquare-kilometer region and frequently traveled the same or similar paths. We confirmed our findings with the ecologists from our team, who study deer movement behavior. Earlier research $[37,38]$ in ecology has shown that the roaming range of deer is typically within two to four square kilometers. This provides us with high confidence about the correctness of the location and sequential movement data we have collected.

We have investigated their movements using the GPS tracking information collected from the five collars placed on the white-tailed deer. Four of the deer stayed within a 


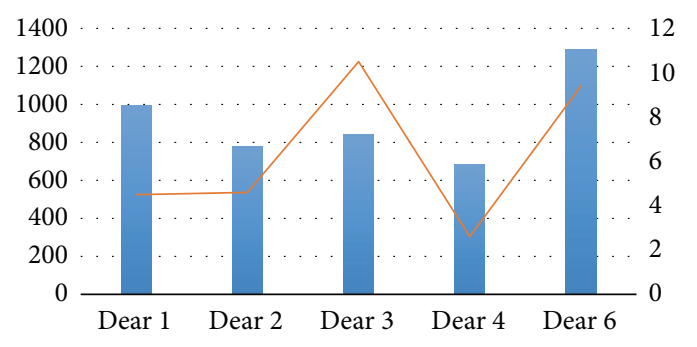

Daily walking average (meters)

_ Maximum walking range $(\mathrm{km})$

FIGURE 10: Daily walking average and maximum walking range of five collared deer.

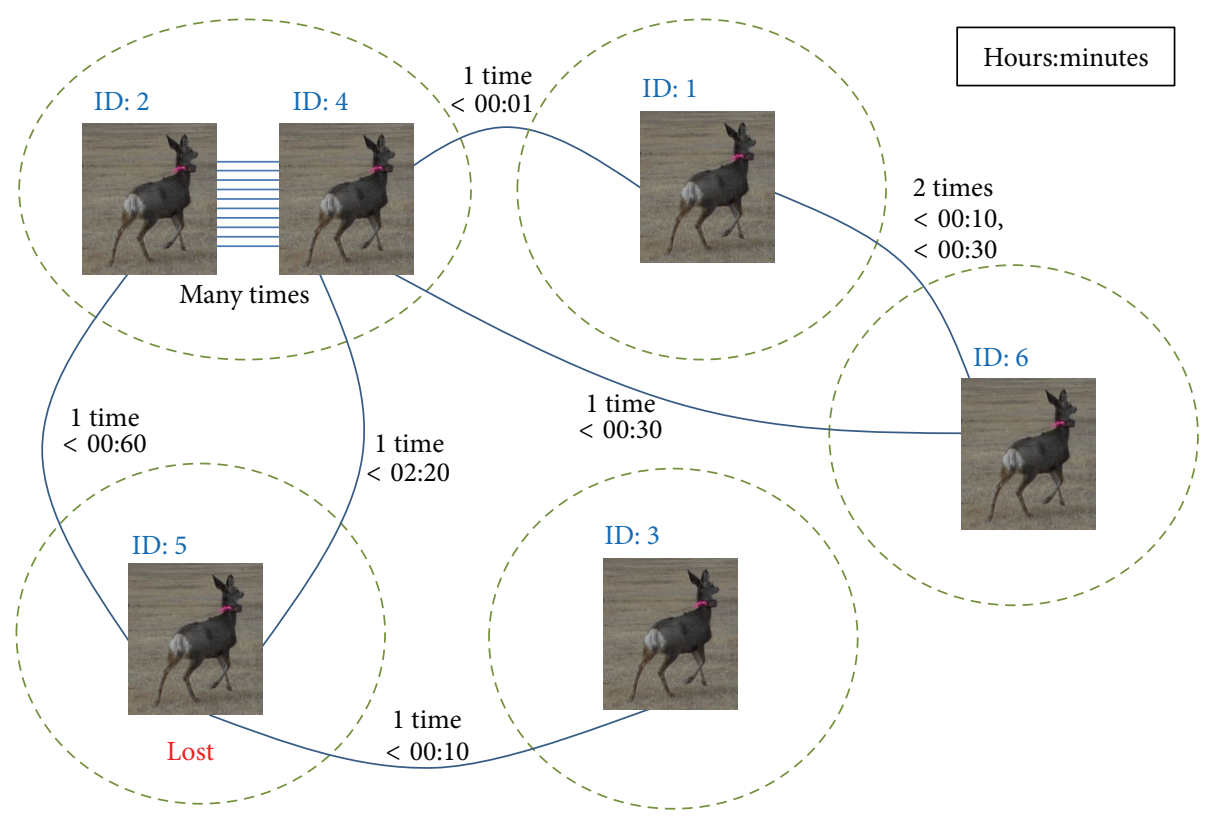

FIGURE 11: Interaction between deer and among deer groups within one month.

10-kilometer range as referred in [1] during the experiment period as shown in Figure 10. The five deer traveled the following distances: $4.5 \mathrm{~km}, 4.6 \mathrm{~km}, 10.5 \mathrm{~km}, 2.6 \mathrm{~km}$, and $9.4 \mathrm{~km}$. Only deer number 3 walked over 10 kilometers because it was trying to find its original living location where it was seen by the helicopter as shown in Figure 9. When considering this, all of the deer stayed in a 10-kilometer range. We have also analyzed their daily walking distance on average as shown in Figure 10. Our research showed they walked a short distance (993 m, $776 \mathrm{~m}, 840 \mathrm{~m}, 684 \mathrm{~m}$, and $1290 \mathrm{~m}$ ) per day on average during the winter season compared with a range between 506 and 1500 meters found in existing research [2]. Therefore, we concluded that our system operates sufficiently to obtain valid results to track the movements of white-tailed deer.

5.1.2. Deer Interactions. Figure 11 shows a diagram of the interactions among the six deer using the interaction data we recorded using the RF-radio-based communication. The deer collars that were within range of the low-power radios, about
$30 \mathrm{~m}$, shared data with each other by sending two beacon signals every 5 seconds within 20-second intervals. Once two deer located each other, they shared their interaction data every 6 seconds. Thus, our system was designed for neighboring deer to share their DTN information at a granularity of less than one minute of interaction duration. Interactions and interaction time durations between two deer in the same group and among deer from different groups are shown in this figure.

As we can clearly see from this figure, deer 2 and deer 4 were a social group as they interacted quite extensively during the experiment period. The identification of the social group (deer 2 and 4 ) is confirmed by the team that captured deer for our experiments (as described in Section 4). The team confirmed that two of the deer captured were from the same herd and the other four were from different herds. Our interaction analysis as shown in Figure 11 confirms that the two deer from the same herd interacted regularly and frequently with each other. The other four deer, captured from different herds, interacted only once or twice with each other. 


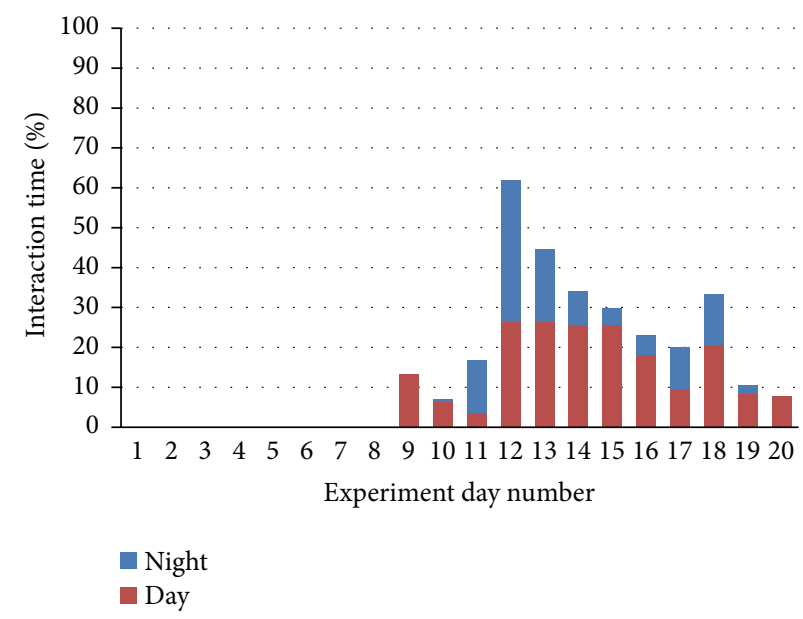

FiguRE 12: Interaction between the two deer from the same herd.

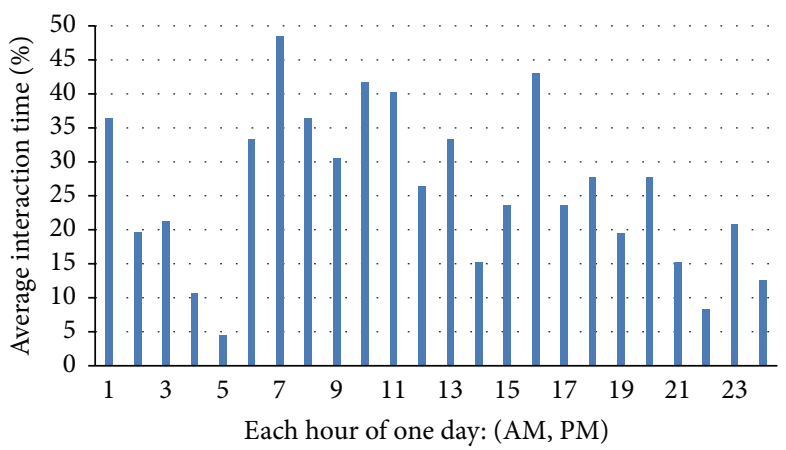

FIGURE 13: Average interaction time for each hour per day.

The deer interaction time duration for the deer from different herds ranged from one minute to 2 hours and 20 minutes.

In particular, we note that our fine-grained sampling was able to capture the brief interaction between deer 1 and 4, which occurred only once and lasted for less than one minute. Such an interaction would have been missed by all of the other wildlife tracking and monitoring systems that we reviewed in related work.

5.1.3. Social Group. Delving more deeply to understand the behavior of a single social group, we analyzed the full 20 days of interactions between deer 2 and 4 who were captured at the same time from the same herd. Figure 12 shows the interaction times of the two deer. After the two captured deer were collared and then released back into the wild, they could not find each other for eight days. Although they were released within five minutes of each other, they were likely disoriented after being sedated for collaring and examination, and so they ran off in different directions. After day 8, they located each other. The interaction data, shown in Figure 12, reveals that once they located each other, they interacted with each other on average $25 \%$ of the time per day for the remaining 12 days. During this period, we have found a 6-percent packet loss by analyzing their interaction data communicated between the motes 2 and 4 . From this data, we can assume that they normally prefer to travel together in the same group but also sometimes maintain their own range while traveling and foraging for food. This figure also shows that the duration of their interaction in a 24-hour day and night period ranged from $8 \%$ to $60 \%$, depending on the day. We classified the interactions as occurring in either the day or night-time within a 24-hour period, by using sunrise and sunset times: approximately $6 \mathrm{AM}$ and $6 \mathrm{PM}$, respectively. We found that they interacted more frequently during the daytime than they did at night: $63.6 \%$ of their interactions occurred during the day and only $36.4 \%$ occurred during night.

We also analyzed the average proportion of time the two deer spent interacting with each other each hour of a 24-hour period, as shown in Figure 13. We found that on average they spent the most time interacting with each other between $6 \mathrm{AM}$ and $1 \mathrm{PM}$. We estimated sunrise to be approximately $6 \mathrm{AM}$, and thus our findings show that the two deer started interacting more frequently right about sunrise, perhaps waking up and starting to forage for food or water. The other noticeable hour period of increased frequency of interaction occurred around 4 PM, which would likely be when they would be grazing and trying to get enough to eat or drink before dark.

We also measured the average proportion of time the two deer spent interacting continuously over the 12-day 


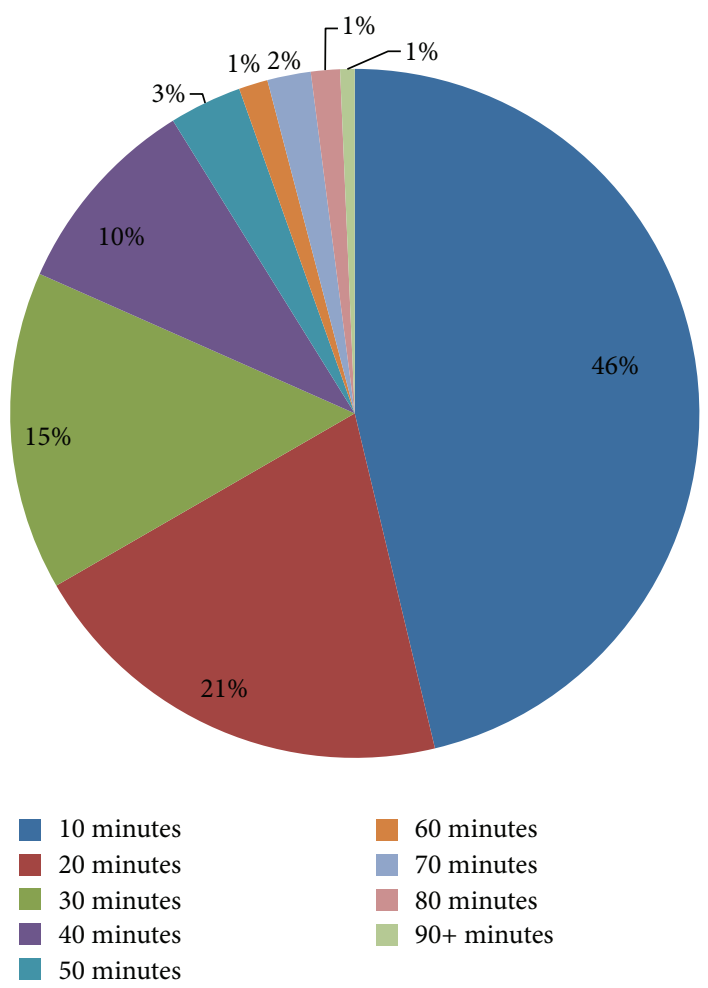

FIGURE 14: Duration of continuous interactions in the same group during the experiment days.

period. Figure 14 shows the average proportion of continuous interaction time between these two deer. Almost half of their total interaction time, $46 \%$, lasted for up to 10 minutes. The majority of the other half (46\%) of their interaction time lasted between 10 and 40 minutes: $21 \%$ between 10 and 20 minutes, $15 \%$ between 20 and 30 minutes, and $10 \%$ between 30 and 40 minutes. The remaining $8 \%$ of their continuous interaction time lasted from more than 40 minutes to more than 90 minutes. These observations demonstrate the feasibility of sampling free-range animal interactions in a manner that is both fine grained and low powered.

5.1.4. DTN. We have evaluated the functionality of our DTN implementation to measure and record the interactions among the six deer in our experiment. Whenever two deer came within range of each other, our DTN algorithm directly shared the two deer IDs as well as any other interaction data in the RAM table from other deer encounters. Figure 15 illustrates an example of the interaction data that was collected, stored, and shared among the five deer who originated from different deer herds. The circled number near each deer picture indicates the order in which each of the deer interacted with one another and the order in which deer interaction information was shared.

First, deer 3 and 5 interacted with each other and shared their DTN data. Next, deer 1 and 6 interacted with each other and shared their information. Third, deer 4 and 6 interacted and shared their DTN data with each other. This interaction also resulted in deer 4 getting all previous interaction data collected by deer 6 , namely, deer 1's contact information.
Fourth, deer 4 and 5 interacted and shared their data with each other. Thus, by this fourth encounter, node 4 had collected and recorded all the DTN interaction data from the 5 "different-herd" deer's previous encounters: from deer 1, 3, 5 , and 6. All of deer 4's collected interaction data was equally shared with deer 2, because these two deer originated from the same herd and were in frequent contact with each other throughout the duration of this experiment.

5.1.5. Fault Tolerance. First, we observe that our system exhibited one type of fault tolerance in terms of how it recorded RF-based proximity contacts. Recall that deer 1 and 4 interacted only once for less than one minute. Upon closer inspection, we found that deer 1 did not collect this interaction data. However, deer 4's low-power radio did successfully collect this interaction data. Such an asymmetric interaction could occur for a variety of reasons: the brevity of the interaction such that there was not enough time to exchange both beacons and/or DTN tables; the asymmetry of wireless channels; and the mobility of the animals. Regardless, our system is able to capture the interaction as long as one of the radios is able to hear the other radio.

More importantly, our system was able to recover some location and interaction information from deer 5 even though we were never able to retrieve its collar. Based on our DTN algorithm for sharing interaction data, we were able to recover enough information from other nodes to be able to calculate the possible area in which deer 5 might have been lost. Deer 3 and 4 had recorded interaction data with node 5 in the beginning and the middle of the experiment 


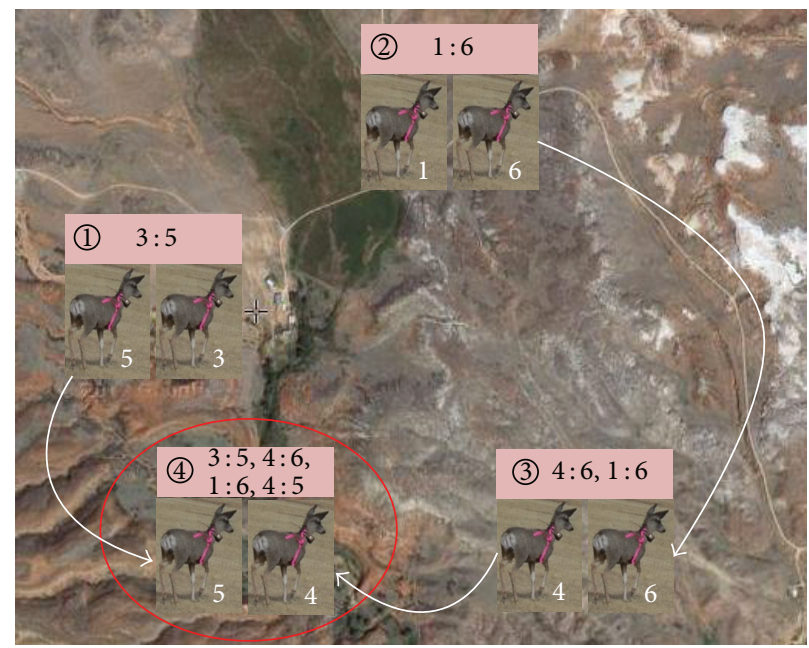

FIGURE 15: Sharing of DTN data among deer originating from different herds.

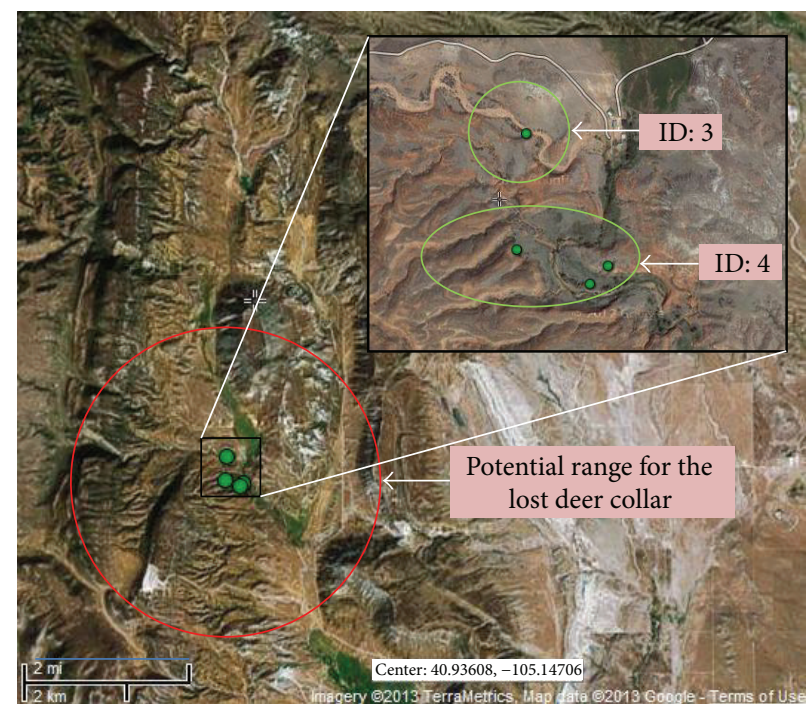

FiguRE 16: Area range of lost deer collar node's possible location.

period. After that time, no further data from deer 5 was collected or recorded. Figure 16 shows four GPS location measurements of deer 5 recorded by deer 3 and 4 at different times. From these measurements, and knowing that deer usually travel within four-square-kilometer region, we can predict the lost collar's potential location area range and map out this area by starting at these GPS coordinates and drawing a 2-kilometer radius circle around these points. The area itself was too rugged to explore to verify this prediction, but this demonstrates the utility of the DTN in recovering otherwise lost data.

A key result from this research experiment was that the DTN algorithm successfully implemented fault tolerance in the wild. Namely, the DTN's sharing of data among nodes that are close enough in proximity to communicate with each other enabled us to collect data from a lost collar that would otherwise have been unavailable. Our system was able to collect deer 5 's interactions through the data shared with deer 2, 3, and 4 (again, see Figure 11).

5.2. Hardware Robustness. As mentioned, we deployed six collars and retrieved five of them. All five retrieved collars functioned and operated flawlessly throughout the 30-day experiment period. Although the area in which we deployed our system experienced considerable snow and wind with temperatures ranging from below zero degree Fahrenheit to about 60 degrees Fahrenheit during the experiment period, all five collars and hardware cases remained intact and protected the MICAz nodes and GPS receivers as well as batteries. No leakage or structural design problems were 
encountered. This demonstrates that our hardware was sufficiently robust.

5.3. False Positives and False Negatives. We have analyzed the location and interaction data we collected to detect if there are any false positives or false negatives. For location data, for each deer, we carefully observed each GPS location recorded and constructed a movement path based on the time when those locations were recorded. Since our GPS sampling rate was once every hour, we checked if the distance between any two consecutive locations is reasonable; that is, a deer can generally cover that distance in one hour. For interaction data, we cross-checked each recorded interaction with the location data of the corresponding deer at about the same time when the interaction was recorded. Using this methodology, we did not find any false positives or false negatives in the location data and neither did we find false positives in the interaction data. We could not calculate false negatives in the interaction data, as we do not have any ground truth of all interactions that happened during the experiment. While location data can provide some information about potential interactions, there is no way to verify that those interactions actually happened.

\section{Discussion}

One feature of WildSense's DTN network that was not exploited in the aforementioned deployment was the ability to extract information in a more timely manner from the DTN even before collars drop from the deer. This would provide scientists with ongoing information as the experiment was progressing, providing closer to real time insights rather than waiting until the end for the collars to drop off. Having ongoing information may be useful to monitor the health and status of deployed collar nodes, so that, for example, new collar nodes could be strategically deployed during the experiment based on intermediate feedback, or the experiment could be modified in middeployment. We could devise a "capture" node that, if deployed close enough to a collar node, would communicate with that collar node and download its DTN table and logged state information to the extent possible. Such a capture node could be mobile, for example, an airborne drone, or could be statically deployed in strategic locations using domain knowledge, for example, a watering hole frequented by the deer. Though we have built software for such a capture node, we have not fully matured this functionality within our DTN system.

The challenges in deploying this DTN system in the wild and retrieving useful data from the deployment were considerable and incurred substantial expense in terms of manpower, time, and funding. As a result, even though we desired to conduct further experiments to improve the amount of data harvested from WildSense, such as by adjusting parameters of the DTN protocol and system or by increasing the duration of the experiment, it was beyond our resources to conduct further deployments in this time frame. We plan to make our software and hardware designs available as open source so such future deployments may benefit from our work and we are also considering options to transfer the WildSense technology to a commercial company.

\section{Conclusions}

In this research, we have described in detail how we developed, deployed, and evaluated a new system for monitoring the movement patterns and interaction behaviors of freerange deer in a rugged, wilderness environment. Our system differs from present-day GPS-only collar systems used for monitoring wildlife by incorporating a low-cost low-power RF-radio mote with each collar, thereby enabling sampling that is both fine grained and long lived. We successfully deployed our WildSense system for 30 days in the Soapstone Prairie Natural Area near Livermore, Colorado, on six wild deer. WildSense recorded proximity-based contacts among deer by using the low-power mote-based radios and implemented a delay-tolerant networking (DTN) protocol to exchange contact information among deer collars. Our system was able to report detailed findings regarding deer behavior and social groupings. Further, WildSense demonstrated the fault tolerance property of DTN protocols; namely, through the propagation of contact information among other collar nodes, we were able to retrieve substantial information from a lost collar node.

\section{Competing Interests}

The authors declare no competing interests.

\section{Acknowledgments}

This work was supported by a grant from the National Science Foundation, IDBR 0754832.

\section{References}

[1] T. W. Grovenburg, J. A. Jenks, R. W. Klaver, C. C. Swanson, C. N. Jacques, and D. Todey, "Seasonal movements and home ranges of white-tailed deer in north-central South Dakota," Canadian Journal of Zoology, vol. 87, no. 10, pp. 876-885, 2009.

[2] G. R. Karns, R. A. Lancia, C. S. DePerno, and M. C. Conner, "Investigation of adult male white-tailed deer excursions outside their home range," Academic Journal, vol. 10, pp. 1-39, 2011.

[3] E. M. Schauber, C. K. Nielsen, L. J. Kjær, C. W. Anderson, and D. J. Storm, "Social affiliation and contact patterns among whitetailed deer in disparate landscapes: implications for disease transmission," Journal of Mammalogy, vol. 96, no. 1, pp. 16-28, 2015.

[4] Z. Zhang, "Routing in intermittently connected mobile ad hoc networks and delay tolerant networks: overview and challenges," IEEE Communications Surveys and Tutorials, vol. 8, no. 1, pp. 24-37, 2006.

[5] K. Fall and S. Farrell, "DTN: an architectural retrospective," IEEE Journal on Selected Areas in Communications, vol. 26, no. 5, pp. 828-836, 2008.

[6] R. D’Souza and J. Jose, "Routing approaches in delay tolerant networks: a survey," International Journal of Computer Applications, vol. 1, pp. 8-14, 2010. 
[7] M. Liu, Y. Yang, and Z. Qin, "A survey of routing protocols and simulations in delay-tolerant networks," in Wireless Algorithms, Systems, and Applications, Y. Cheng, D. Y. Eun, Z. Qin, M. Song, and K. Xing, Eds., vol. 6843 of Lecture Notes in Computer Science, pp. 243-253, Springer, New York, NY, USA, 2011.

[8] T. Spyropoulos, K. Psounis, and C. S. Raghavendra, "Spray and wait: an efficient routing scheme for intermittently connected mobile networks," in Proceedings of the ACM SIGCOMM Workshop on Delay-Tolerant Networking (WDTN '05), pp. 252259, ACM, Philadelphia, Pa, USA, 2005.

[9] Q. Li, S. Zhu, and G. Cao, "Routing in socially selfish delay tolerant networks," in Proceedings of the IEEE INFOCOM 2010, pp. 1-9, IEEE, San Diego, Calif, USA, March 2010.

[10] B. Pásztor, M. Musolesi, and C. Mascolo, "Opportunistic mobile sensor data collection with SCAR," in Proceedings of the IEEE Internatonal Conference on Mobile Adhoc and Sensor Systems (MASS '07), 12, 1 pages, Pisa, Italy, October 2007.

[11] W. Zhao, M. Ammar, and E. Zegura, "A message ferrying approach for data delivery in sparse mobile ad hoc networks," in Proceedings of the 5th ACM International Symposium on Mobile Ad Hoc Networking and Computing (MoBiHoc '04), pp. 187-198, ACM, Tokyo, Japan, May 2004.

[12] K. C. Lee, U. Lee, and M. Gerla, "Survey of routing protocols in vehicular ad hoc networks," in Advances in Vehicular AdHoc Networks: Developments and Challenges, pp. 149-170, IGI Global, 2010.

[13] P. Zhang, C. M. Sadler, S. A. Lyon, and M. Martonosi, "Hardware design experiences in ZebraNet," in Proceedings of the 2nd International Conference on Embedded Networked Sensor Systems (SenSys '04), pp. 227-238, ACM, Baltimore, Md, USA, November 2004.

[14] T. Liu, C. M. Sadler, P. Zhang, and M. Martonosi, "Implementing software on resource-constrained mobile sensors: experiences with Impala and ZebraNet," in Proceedings of the 2nd International Conference on Mobile Systems, Applications and Services (MobiSys '04), pp. 256-269, ACM, Boston, Mass, USA, June 2004.

[15] P. Juang, H. Oki, Y. Wang, M. Martonosi, L. S. Peh, and D. Rubenstein, "Energy-efficient computing for wildlife tracking: design tradeoffs and early experiences with ZebraNet," ACM SIGOPS Operating Systems Review, vol. 36, no. 5, pp. 96-107, 2002.

[16] Y. Wang, P. Zhang, T. Liu, C. Sadler, and M. Martonosi, "CRAWDAD data set princeton/zebranet (v. 2007-02-14)," 2007, http://crawdad.cs.dartmouth.edu/princeton/zebranet.

[17] T. Wark, W. Hu, P. Sikka et al., "A model-based routing protocol for a mobile, delay tolerant network," in Proceedings of the 5th International Conference on Embedded Networked Sensor Systems (SenSys '07), pp. 421-422, ACM, Sydney, Australia, November 2007.

[18] M. Rutishauser, V. V. Petkov, J. Boice et al., "CARNIVORE: a disruption-tolerant system for studying wildlife," in Proceedings of the 19th International Conference on Computer Communications and Networks (ICCCN '10), pp. 1-8, IEEE, Zurich, Switzerland, August 2010.

[19] A. Pentland, R. Fletcher, and A. Hasson, "DakNet: rethinking connectivity in developing nations," Computer, vol. 37, no. 1, pp. 78-83, 2004.

[20] Wizzy Project, http://www.oocities.org/township_transport/ courier.html.

[21] T. V. Prabhakar, S. Nambi, A. U. n et al., "A novel DTN based energy neutral transfer scheme for energy harvested WSN
Gateways," ACM SIGMETRICS Performance Evaluation Review, vol. 38, no. 3, pp. 71-75, 2011.

[22] X. Zhang, J. Kurose, B. N. Levine, D. Towsley, and H. Zhang, "Study of a bus-based disruptiontolerant network: mobility modeling and impact on routing," in Proceedings of the 13th Annual ACM International Conference on Mobile Computing and Networking (MobiCom '07), pp. 195-206, New York, NY, USA, 2007.

[23] J. Su, A. Goel, and E. de Lara, "An empirical evaluation of the student-net delay tolerant network," in Proceedings of the Annual International Conference on Mobile and Ubiquitous Systems, pp. 1-10, 2006.

[24] P. Hui, A. Chaintreau, J. Scott, R. Gass, J. Crowcroft, and C. Diot, "Pocket switched networks and human mobility in conference environments," in Proceedings of the ACM SIGCOMM Workshop on Delay-Tolerant Networking (WDTN '05), pp. 244-251, ACM, Philadelphia, Pa, USA, August 2005.

[25] R. N. Handcock, D. L. Swain, G. J. Bishop-Hurley et al., "Monitoring animal behaviour and environmental interactions using wireless sensor networks, GPS collars and satellite remote sensing," Sensors, vol. 9, no. 5, pp. 3586-3603, 2009.

[26] P. Sikka, P. Corke, and L. Overs, "Wireless sensor devices for animal tracking and control," in Proceedings of the 29th Annual IEEE International Conference on Local Computer Networks, pp. 446-454, IEEE, Tampa, Fla, USA, November 2004.

[27] Y. Guo, P. Corke, G. Poulton, T. Wark, G. Bishop-Hurley, and D. Swain, "Animal behaviour understanding using wireless sensor networks," in Proceedings of the 31st Annual IEEE Conference on Local Computer Networks (LCN '06), pp. 607-614, Tampa, Fla, USA, November 2006.

[28] B. Thorstensen, T. Syversen, T. A. Bjørnvold, and T. Walseth, "Electronic shepherd-a low-cost, low-bandwidth, wireless network system," in Proceedings of the 2nd International Conference on Mobile Systems, Applications, and Services (MobiSys '04), pp. 245-255, ACM, Boston, Mass, USA, June 2004.

[29] V. Dyo, S. A. Ellwood, D. W. MacDonald et al., "Evolution and sustainability of a wildlife monitoring sensor network," in Proceedings of the 8th ACM International Conference on Embedded Networked Sensor Systems (SenSys '10), pp. 127-140, ACM, Zurich, Switzerland, November 2010.

[30] D. Anthony, W. P. Bennett, M. C. Vuran et al., "Sensing through the continent: towards monitoring migratory birds using cellular sensor networks," in Proceedings of the ACM/IEEE 11th International Conference on Information Processing in Sensor Networks (IPSN '12), pp. 329-340, Beijing, China, April 2012.

[31] V. R. Jain, R. Bagree, A. Kumar, and P. Ranjan, "wildCENSE: GPS based animal tracking system," in Proceedings of the International Conference on Intelligent Sensors, Sensor Networks and Information Processing (ISSNIP '08), pp. 617-622, Sydney, Australia, December 2008.

[32] Crossbow MICAz datasheet, http://www.openautomation.net/ uploadsproductos/micazdatasheet.pdf.

[33] B. Kusy, P. Dutta, P. Levis, M. Maroti, A. Ledeczi, and D. Culler, "Elapsed time on arrival: a simple and versatile primitive for canonical time synchronisation services," International Journal of Ad Hoc and Ubiquitous Computing, vol. 1, pp. 239-251, 2006.

[34] Crossbow MTS420 Sensor Board, http://tinyos.stanford.edu/ tinyos-wiki/index.php/MTS420.

[35] Texas instruments TPS61201 Low input voltage synchronous boost converter with 1.3-a switches, 2007, http://www.ti.com/ product/TPS61201. 
[36] Advanced Telemetry Systems, https://atstrack.com/.

[37] R. A. Garrott, G. C. White, R. M. Bartmann, L. H. Carpenter, and A. W. Alldredge, "Movements of female mule deer in northwest Colorado," Journal of Wildlife Management, vol. 51, no. 3, pp. 634-643, 1987.

[38] P. Beier and D. R. McCullough, "Factors influencing whitetailed deer activity patterns and habitat use," Wildlife Monographs, vol. 109, pp. 3-51, 1990.

[39] K. E. Lagory, "Habitat, group size, and the behaviour of whitetailed deer," Behaviour, vol. 98, no. 1, pp. 168-179, 1986.

[40] Soapstone Prairie Natural Area, 2007, http://m.fcgov.com/ naturalareas/pdf/soapstone-management-plan.pdf.

[41] T. Hobbs, "IDBR: WildSense: instrumenting wildlife to gather contact rate information using delay tolerant wireless sensor networks," IACUC Protocol 11-2503A, 2011. 


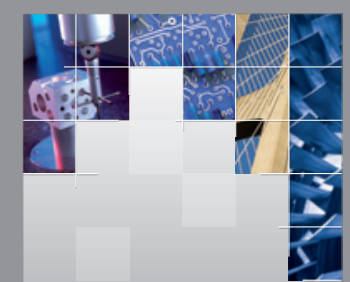

\section{Enfincering}
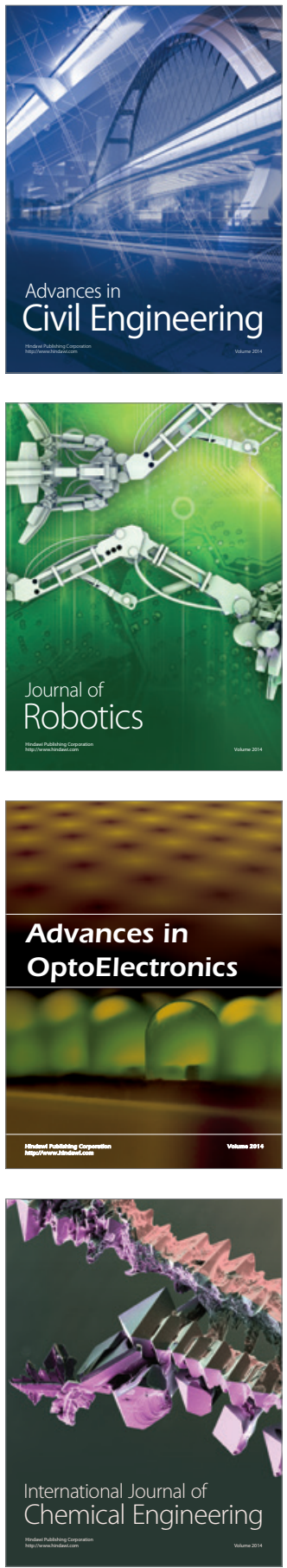

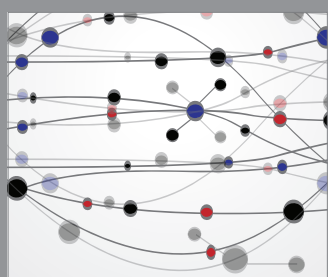

The Scientific World Journal

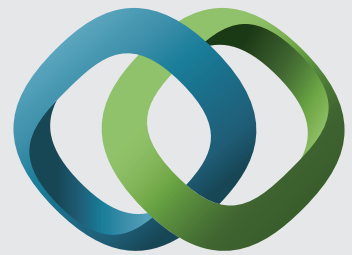

\section{Hindawi}

Submit your manuscripts at

http://www.hindawi.com
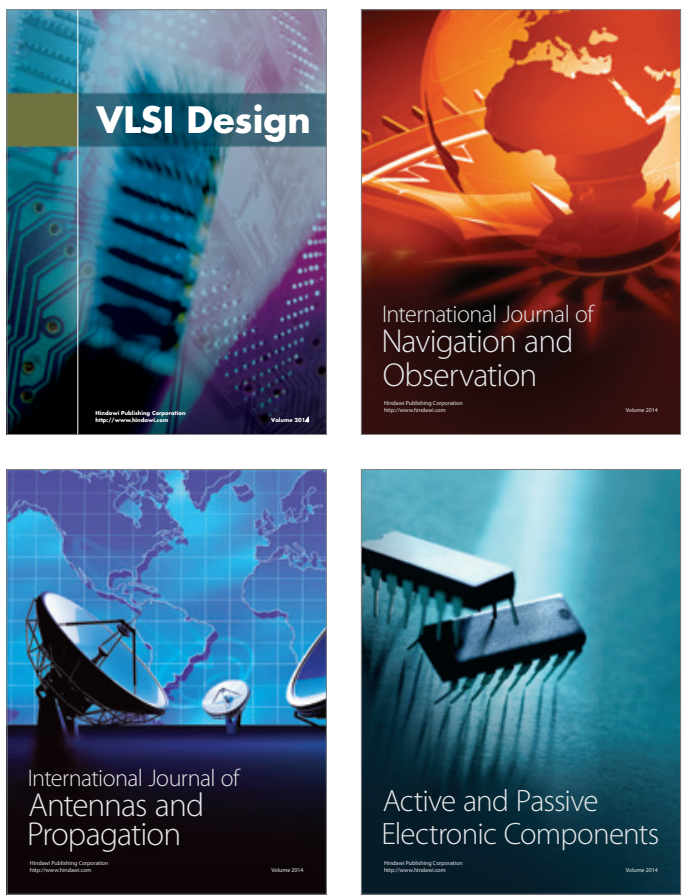
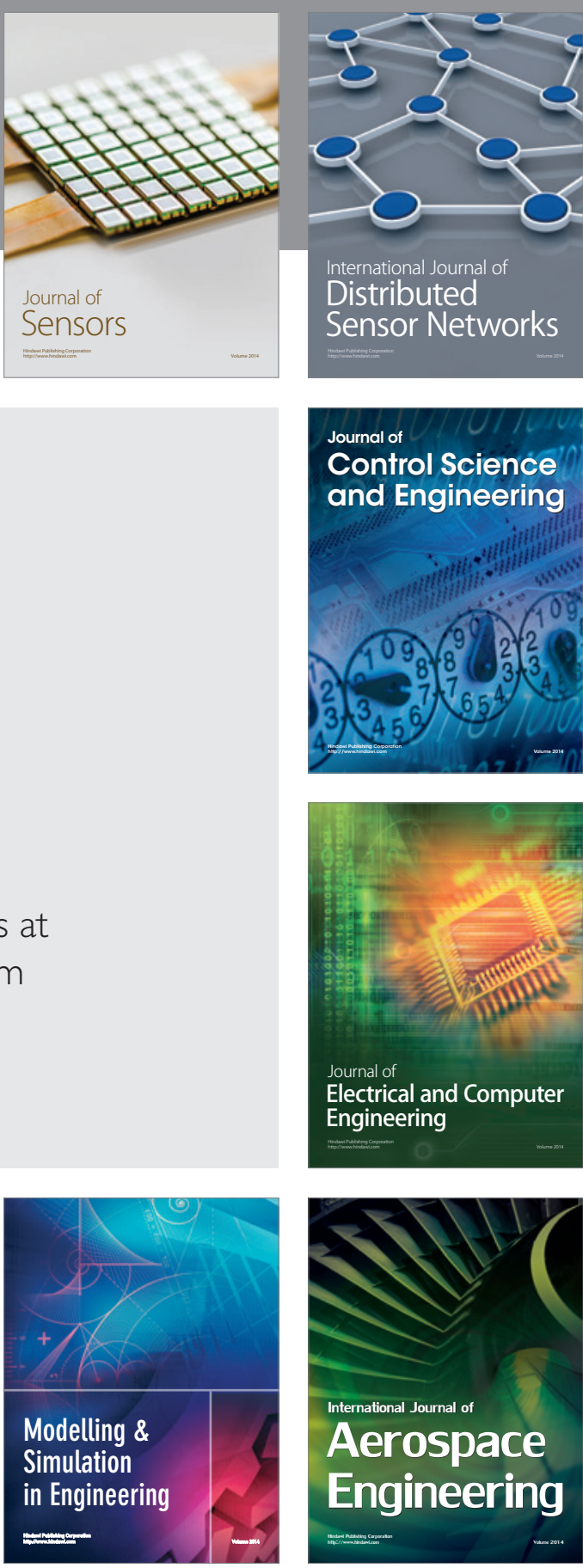

International Journal of

Distributed

Sensor Networks

Journal of

Control Science

and Engineering
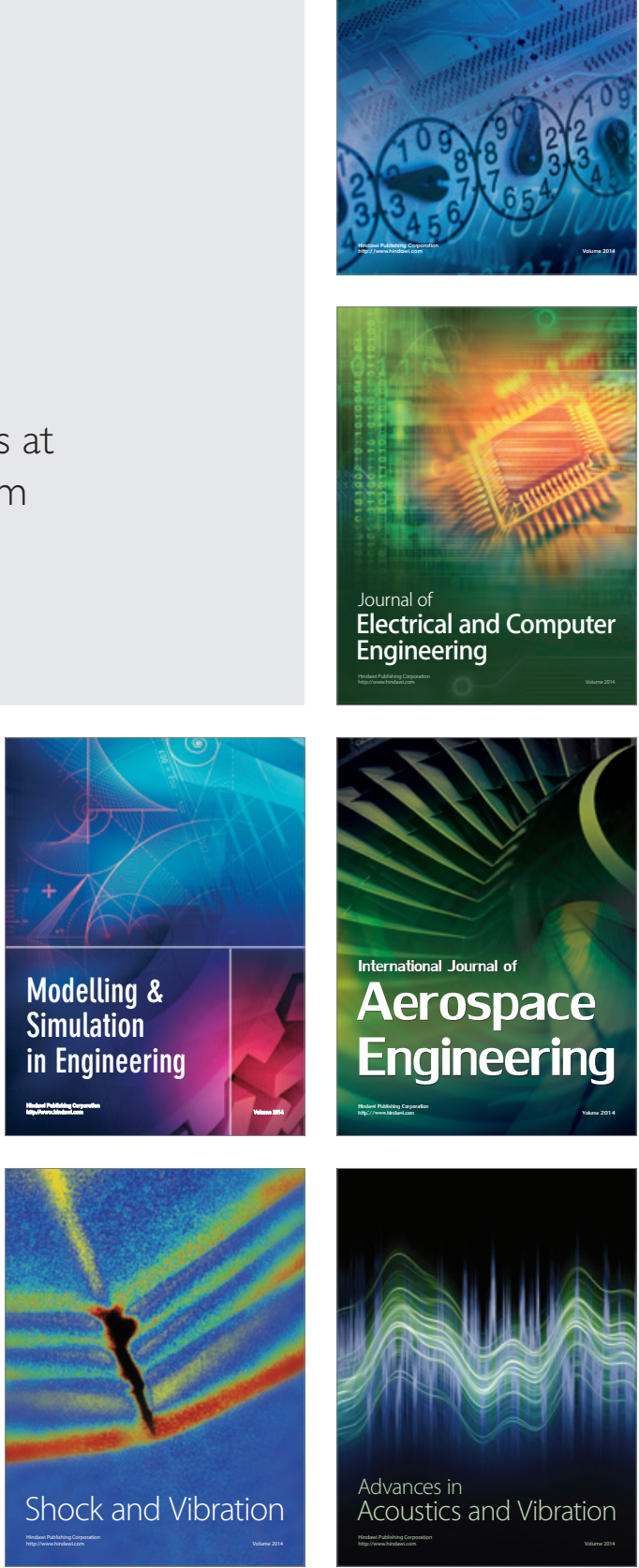